\title{
A SURVEY ON CBIR TECHNIQUES AND LEARNING ALGORITHM COMPARISON
}

\author{
Pooja Devi ${ }^{1}$ and Mahesh Parmar $^{2}$
}

\begin{abstract}
CBIR is the task of retrieving images from huge set of database on the basis of their visual content. For CBIR technology some strong applications could be recognized as architecture design, art \& craft museums, archaeology, medical imaging and geographic info system, trademark databases, weather forecast, image classification, criminal investigations, image search over internet and remote sensing field for indexing biomedical images by contents of this paper provides technical achievements survey in the research CBIR area. This paper tries to provide comprehensive CBIR review.
\end{abstract}

Keywords - CBIR, Application, Learning Algorithms, Techniques.

\section{INTRODUCTION}

Advances in technology for example digital cameras, scanners, storage media and huge online attain have led to a proliferation of both digital images and personal set. Image retrieval has been an exceptionally research discipline over final 10 years, but first evaluation articles on access manner in the image databases appeared already. The essential content established image retrieval approach consists of two predominant materials, feature classification and extraction (Figure 1). The images are kept in a database called as Image Database. After preprocessing, images are segmented through applying the technique defined. CBIR method consists of two predominant steps. The first one is the characteristic extraction; the place a collection of function is generated to symbolize content of each and every image. The second is similarity size where a distance between question image and all image in database is computed making use of their feature vectors. The work serious about making use of low degree aspects similar to color, texture, shape and spatial design for image representation. The earliest use of the term CBIR in literature seems to have been used through authors in to define their experiments of automatic images retrieval from a database through shape and color features.

There are four images retrieves phases from the database based on geometric properties of the input query images boundary [1].

- $\quad$ Image database generation phase

- Input images from scanner, thinning editing's

- $\quad$ Outline based image retrieval phase

- $\quad$ Identify global shape similarity

\footnotetext{
${ }^{1}$ Research scholar, M.Tech(Information Technology) Madhav Institute of Technology and Science Gwalior, Madhya Pradesh, India

${ }^{2}$ Assistant Professor (Dept. of CSE \& IT) Madhav Institute of Technology and Science Gwalior, Madhya Pradesh, India
} 


\section{LITERATURE SURVEY}

By MVHAMMAD HAMMAD ME MON et.al.[5] present that present days data retrieval systems get additional attention because of the growing multimedia technologies use. This paper demonstrates an image retrieval system based on multiple regions that provide a client interface for helping to identify watershed regions-of-interest inside of an input image. The relationship between semantic ideas and visual elements is established through supervised Bayesian learning from positive bags. On standard datasets the proposed algorithm has been using and accomplishes great annotation performance [5].

By Pushpalatha S. Nikkam et.al.[6] present that CBIR is a procedure to found a desired image from a substantial database. A template for shape based hierarchical feature matching method for CBIR system. It uses a combination of global feature for shape based templates. In this work a novel learning technique is put forth which is based on hierarchal data decomposition. The proposed technique founds learning algorithm where feature extraction procedure is executed to detect edge, orientations and dataset images shape. The classification of dataset is taken care through support vector machine algorithm with the $99.09 \%$ accuracy. The retrieval outcomes of proposed model are illustrated in terms of precision and recall, enhanced efficacy of retrieval is compared to other present models [6].

By CHAWKI Youness et.al.[7]present retrieval systems are typically based on the key words for image search, but in numerous case it cannot meet demands for various user with numerous view. A new CBIR method is presented, which is based on image frequency content. Indeed, we have used the 2-D ESPRIT technique to mine from image the frequency content for constructing the vector descriptor. This method is applied to the Coil_100 database and experimental outcomes present that this approach increases the image retrieval precision [7].

By Ekta Gupta et.al.[8] present that CBIR uses visual image contents for example global features-color feature, shape feature, texture feature, and local features-spatial domain present to indicate and image index. CBIR technique combines global and local features. In this paper for classification procedure, SVM used. The experimental outcomes present enhanced outcomes in comparison to earlier approach. In this paper, proposed a calculation which consolidates the advantages of some various calculations to enhance the exactness and execution of recovery [8].

By KAMLESH KUMAR et.al.[9] present since last few years, CBIR system has got additional attention from its generic to particular use. CBIR depends upon visual low-Ievel feature extraction i-e color, texture, shape and spatial layout. In this paper, LBP has been employed for texture and image analysis and also it is compared with average RGB color image descriptor technique. And then a complementary feature extraction method applying average RGB color and LBP texture technique has been proposed for CBIR. Euclidean distance is used as similarity measure for finding similar images in the database. The experimental outcomes are created applying MATLAB. The obtained outcomes proved that accuracy and efficiency of proposed technique in terms of overall precision, recall, measure and retrieval time are quite enhance than single color and texture feature extraction method [9].

By Vrushali A. Wankhede et.al.[10] present that Content-based video retrieval is most interesting point where it can be used in our daily life. Video retrieval is regarded as one of the 
most important in multimedia research. The development of multimedia data kind there is demand of video retrieval system. Video retrieval can be used for video search and browsing which are valuable in web applications. Selection of extracted features performs an important role in content based video retrieval. The main goal of this paper is that, user can give the two different types of input in the form of image query and the text query. First one is that provide the input in form of image query and retrieved image which is similar to the query image through applying CBIR algorithm. In this paper provide a detail description of a system developed for retrieving images similar to a query image from a various huge image set. Second one is that provide the input in the form of text query and retrieved image through applying ABIR method. Annotation is never complete [10]

By Radu Andrei Stefan et.al.[11] presents that a study on the effectiveness of hierarchical clustering techniques application and classification for imaging context in CBIR. The study has the purpose to compare obtained outcomes from applying various hierarchical clustering algorithms with numerous input parameters and configurations. The aims are also to highlight performance improvements and the costs brought up through integration of such approach in the CBIR [11].

By Katta Sugamya et.al. [13] present that This paper proposes a new two-step strategy in which first step is feature extraction using low level features (color, shape and texture) while SVM classifier is used in the second step to handle the noisy positive examples. Thus, an efficient algorithm of image retrieval based on color-correlogram for color feature extraction, wavelet transformation for extracting shape features and Gabor wavelet for texture feature extraction is proposed. Further, multiple features and different distance metrics are combined to obtain image similarity using SVM classifier. Results based on this approach are found encouraging in terms of color, shape and texture image classification accuracy [13].

By Syntyche Gbèhounou et.al. [14] present that On this paper, decide upon most likely of low stage facets used in CBIR particularly these centered $\mathrm{n}$ SIFT descriptors. To do not forget difficult emotion belief procedure, here don't forget color and texture features and one international scene descriptor: GIST. Supposed the select features could implicitly encrypt highlevel data about emotions because of their accuracy in the different CBIR applications [14].

By Shu Wang et.al. [15] a systematic method to reduce appearance SBIR gap. In this paper, sketches and extracted edges are treated as set of line segments, laying the foundation for better sketch edge description and noise impact reduction from a new angle. Object boundary selection algorithm is used to reduce the impact of noisy edges. In this paper, spatial constraint and coherent constraint are proposed to filter the false matches that degrade retrieval performance [15].

By K. Bozas et.al. [16] present that identified similarities between a hand drawn sketch and the natural images in a database. They used voting process to detect duplicate shape and structure patches. Local Sensitive Hashing technique is well suited for estimating the similarity between sets. In this system, impact of noisy edges in the image degrades the retrieval performance [16]. 
By R. Zhou et.al. [17]proposes a sketch based algorithm for large scale image retrieval and practical prototype system is develop to search result from millions of images. Two candidate regions are used feature extraction, firstly orientation features are extracted and then these features are organized in a hierarchal way to generate global-to-local features. Therefore, hierarchical database index is structured and then image can be retrieved among large scale image database online. This system filters out a large number of irrelevant images quickly [17].

By M. Eitz et.al. [18]defined general benchmark for evaluating the performance of any SBIR system. For matching pictures SBIR system uses the information contained in the designed 3D shape. M. Eitz, K. Hildebrand, T. Boubekeur et al., introduces Spark feature for specialized SBIR in a Bag of Visual Words (BoVW) framework, GF-HOG is an image descriptor suitable for Sketch based Image Retrieval (SBIR). It treats sketches edges as a set of points, rather than describing image patches, describe lines and their relationships to extract image from dataset. It is much slower patch correlation based approach of Self-Similarity [18].

III. CBIR based on Visual Contents

Here we have various approaches of retrieving images from large database on the basis of its visual contents and some of its limitations [12].

Table -1

\begin{tabular}{|l|l|l|l|}
\hline No. & Features & Approaches & Limitations \\
\hline 1 & Colour & $\begin{array}{l}\text { Histogram and } \\
\text { colour moments }\end{array}$ & $\begin{array}{l}\text { Query image is an unknown image, then the } \\
\text { retrieval performance is poor }\end{array}$ \\
\hline 2 & Colour & Region Histogram & $\begin{array}{l}\text { Outcomes within the mismatch of the retrieval } \\
\text { method when the image orientation, and } \\
\text { position or scales are } \\
\text { Altered. }\end{array}$ \\
\hline 3 & Colour & Colour Histogram & $\begin{array}{l}\text { Similarity measure is elevated to retrieve the } \\
\text { texture areas from a database of common pics. }\end{array}$ \\
\hline 5 & Shape & Fourier Transform & $\begin{array}{l}\text { Semantically relevant } \\
\text { images will be retrieved with amount of time }\end{array}$ \\
\hline 6 & Shape & $\begin{array}{l}\text { Curvature } \text { Scale } \\
\text { and the query image } \\
\text { information becomes a new cluster }\end{array}$ & $\begin{array}{l}\text { Given a query image, the user has to select the } \\
\text { region of interest from the query imag }\end{array}$ \\
\hline 7 & Shape & Template Matching & $\begin{array}{l}\text { Images from the history of the person access } \\
\text { patterns, and the access frequencies of the } \\
\text { image in the database. }\end{array}$ \\
\hline 8 & Shape & Convex Parts & $\begin{array}{l}\text { The feedback can be got from the user again } \\
\text { and again, till the user is satisfied with the } \\
\text { results. }\end{array}$ \\
\hline 9 & Shape & $\begin{array}{l}\text { Elastic } \\
\text { Deformation of } \\
\text { Templates }\end{array}$ & $\begin{array}{l}\text { The similarity distance is found between the } \\
\text { query image and the images } \\
\text { belonging to the predicted cluster alone. }\end{array}$ \\
\hline Wavelet Transform & \begin{tabular}{l} 
All the classes are assigned to one of the two \\
\hline
\end{tabular} & Texture & Colack \\
\hline 4
\end{tabular}




\begin{tabular}{|l|l|l|l|}
\hline 11 & Texture & Edge Statistics & $\begin{array}{l}\text { possible groups of classes. } \\
\text { distribution of the image data, the k-means } \\
\text { clustering often cannot separate images }\end{array}$ \\
\hline 12 & Texture & Gabor Filters & $\begin{array}{l}\text { The machine learning } \\
\text { predicts the category of the query image }\end{array}$ \\
\hline 13 & Texture & Statistical & $\begin{array}{l}\text { The query image belongs to the class for which } \\
\text { the membership is very large }\end{array}$ \\
\hline
\end{tabular}

\section{CBIR TECHNIQUES}

There is few methods CBIR system used for image retrieval in numerous applications.

\section{A. Relevance Feedback:}

As per time varies many users may have various requirement. User follows the following classical scenario for relevance feedback in the CBIR:

i) Machine provides initial image retrieval outcomes.

ii) User provides his opinion that whether retrieved image is relevant or not.

iii) Machine receives user feedback and again search for images according to user query.

\section{B. Semantic Template:}

This method is created to support high level image retrieval and not so extensively used. This method is typically defined as representative feature of concept calculated from a sample images collection.

\section{Wavelet Transform:}

Wavelet transform is diminutive waves based called as limited duration and variable frequency wavelet. Discrete Wavelet transform distributes images into four various parts namely higher frequency part (HH), High Low Frequency part (HL), Low High Frequency part (LH), and Lower frequency part (LL). After doing vertical parts as 1-level images decomposition, it computes moments of each part and store and use it as feature to find images.

\section{Gabor Filter:}

It is extensively used for texture analysis because of its similar characteristics with human perception. A 2D Gabor function $\mathrm{g}(\mathrm{x}, \mathrm{y})$ consists of a sinusoidal plane wave of some frequency and orientation (Carrier), and 2D translated. Gaussian Envelope is used to modulate it.

\section{E. Support Vector Machine:}

It is supervised learning method in which information is analyzed and identify pattern used for classification purpose. In classification it takes input set, read it and forms output for all desired input and if output is continuous then regression is performed [2].

\section{COMMON FEATURES FOR IMAGE RETRIEVAL}

A feature is described as capturing a various visual image property. In common, image features can be either global or local. The global features describe the visual content of the full image, 
whereas local features define regions or image content objects. The global mining benefit is its high speed for both computing similarity and extracting features. However, global features are often too rigid to represent an image. Local-feature method provides slightly higher retrieval efficient than global features.. While local methods provide additional robust knowledge, they are additional exclusive computationally because of high dimensionality of their feature spaces and typically requirement nearest neighbor's approximation to achieve points matching [3].

\section{A. Color Features}

The color has extensively been used in IR systems, because of its fast and easy computation. Color is also an intuitive feature and performs an important role in the image matching. Color histogram is generally used color feature representation in the image retrieval. The original idea to use histogram for retrieval comes from Swain and Ballard, who realized the power to identify an object applying color, is much higher than that of a gray scale. Although feature of global color is easily to calculate and can provide reasonable discriminating power in the image retrieval. It tends to provide too many false positives when image set is huge. Various research outcomes suggested that applying color layout is an image retrieval higher solution. To extend global color feature to a local one, a natural method is to divide full image into sub-blocks and mine color features from all of the sub-blocks. The advantage of this method is its accuracy while drawback is the usual complex problem of reliable image segmentation.

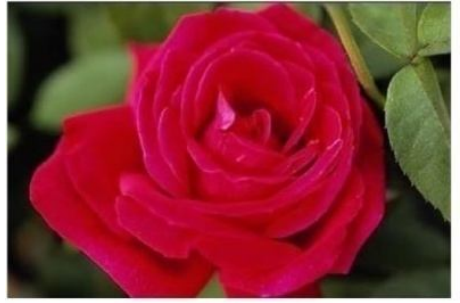

(a)

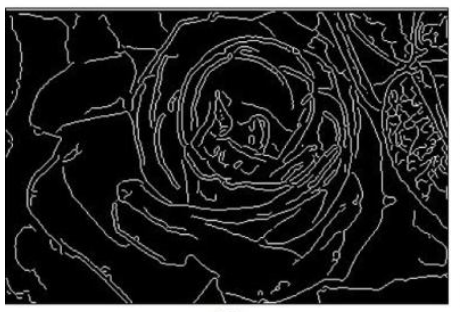

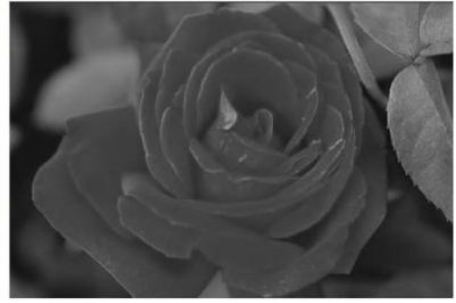

(b)

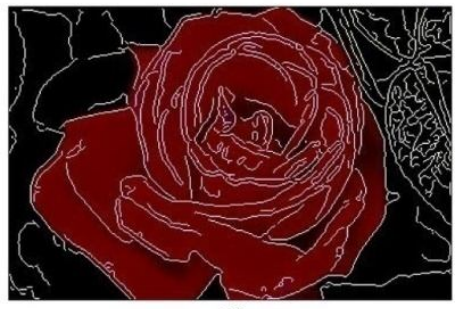

(d)

Figure 1. Color feature in CBIR

\section{B. $\quad$ Texture Features}

Texture is a property that represents the picture surface and constitution. Texture will also be defined as a typical repetition of sample and aspect on a surface. Picture textures are complex visual patterns composed of regions or entities with sub-patterns with traits of brightness, color, form, measurement...and so on. The generally known texture descriptors are Wavelet Transform, Gabor-filter, and Tamura features. 

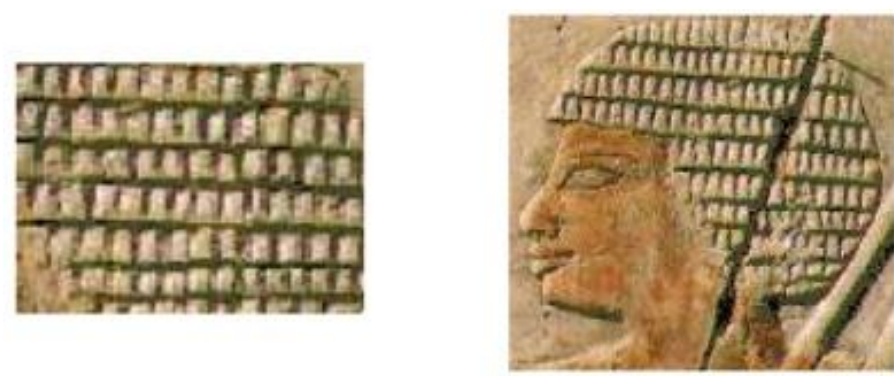

Figure 2. Texture feature in CBIR

\section{C. $\quad$ Shape Features}

Shape can commonly be described as position of object regardless description, orientation, and size.. In the direction of applying shape as feature of an image, it is necessary to object or region boundaries define in the image and this is a challenge.. Since robust and correct image segmentation is complex to achieve, the use of shape features for image retrieval has been limited to special applications where regions or objects are readily available. More commonly, the form representations can also be divided into two classes, boundary-centered that makes use of most effective the outer shape boundary and vicinity-centered that makes use of the entire shape vicinity. Essentially the most effective representatives for these two extraordinary classes are Fourier descriptor and moment invariants.

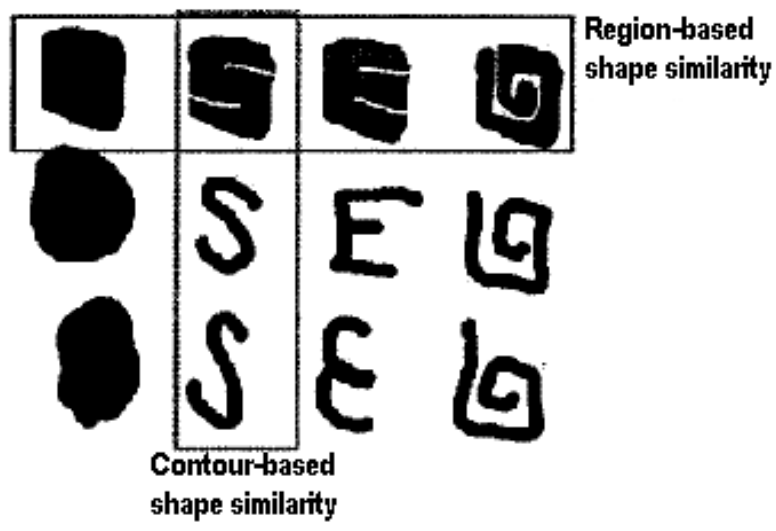

Figure 3. Shape feature in CBIR

\section{D. $\quad$ Spatial Location Features}

Spatial location is also important and is region segmentation used.. Such as, sea and sky may have the same characteristics of color and texture but spatial data is not similar. Sky classically represents above portion whereas sea is at the below image portion. Hence, spatial multiple objects data in image extracts important images retrieval data. Most spatial information is presented in terms of 2D strings. The 2-D representation.

\section{E. $\quad$ Local Image Features}

Local features are small square, sub-images extracted from the original image. They can be considering having two different types:

- The patches: They extracted from the images at salient points and dimensionality reduced using Principal Component Analysis (PCA) transformation. 
- SIFT descriptors: They extracted at Harris interest points. To use local features for image retrieval, three different methods are available:

- Direct transfer: The local elements extracted from each database picture and from the question snapshot. Then, the nearest neighbors for each of the neighborhood facets of the question searched and the database portraits containing all these neighbors returned.

- LFIDM: The local features from the query snapshot in comparison with the neighborhood elements of every image of the database and the distances between them summed up. The images with the lowest total distances are returned.

- Histograms of local features: A reasonably large amount of local features from the database is clustered and then each database image represented by a histogram of indices of these clusters. These histograms are then compared using the Jeffrey divergence.

\section{E. CBIR SySTEM WITH LOW LEVEL FEATURES AND LEARNING ALGORITHM}

Here we have some CBIR system works on low level features of image with their learning algorithms and similarity measures [4].

Table-2

\begin{tabular}{|l|l|l|l|}
\hline $\begin{array}{l}\text { CBIR } \\
\text { System }\end{array}$ & $\begin{array}{l}\text { Low level } \\
\text { features }\end{array}$ & Learning algorithm & Similarity matching \\
\hline RETIN & $\begin{array}{l}\text { 1. Color } \\
\text { 2. Texture }\end{array}$ & $\begin{array}{l}\text { Color histogram } \\
\text { Output of gabor transform }\end{array}$ & $\begin{array}{l}\text { Weighted } \\
\text { minkowski distance }\end{array}$ \\
\hline KIWI & $\begin{array}{l}\text { 1. Color } \\
\text { 2. Shape }\end{array}$ & Color histogram Gabor filters & Euclidean space \\
\hline i PURE & $\begin{array}{l}\text { 1. Color } \\
\text { 2. Texture } \\
\text { 3. Shape } \\
\text { 4. Spatial }\end{array}$ & $\begin{array}{l}\text { average color in CIE's LUV space } \\
\text { Wold decomposition Fourier descriptor } \\
\text { location- centroid }\end{array}$ & \\
\hline
\end{tabular}

\section{VII.CONCLUSION}

Survey paper reviews the fundamental theories of CBIR algorithms and development in this field. These algorithms retrieve digital image from significant image database. Snapshot is retrieved from the low degree visible content elements of query image that's color, texture, form and spatial area. We review the visible content material description of image and then the essential schemes for CBIR are discussed.

\section{REFERENCES}

[1]. R. Rahimzadeh, A. Farzan and Y. Fanid Fathabad," A SURVEY ON SEMANTIC CONTENT BASED IMAGE RETRIEVAL AND CBIR SYSTEMS", International Journal on "Technical and Physical Problems of Engineering” (IJTPE) March 2014, Issue 18, Volume 6, Number 1 Pages 64-69. 
[2]. Priyanka Malode and Prof. S. V. Gumaste," A Review Paper on Content Based Image Retrieval”, International Research Journal of Engineering and Technology (IRJET) Volume: 02 Issue: 09 | Dec-2015

[3]. Mohammed Alkhawlani, Mohammed Elmogy and Hazem El Bakry," Text-based, Content-based, and Semantic-based Image Retrievals: A Survey", International Journal of Computer and Information Technology (ISSN: 2279 - 0764) Volume 04 - Issue 01, January 2015, pp: 58-66.

[4]. Ashwani Kr. Yadav, R. Roy, Vaishali and Archek Praveen Kumar," Survey on Content-based Image Retrieval and Texture Analysis with Applications", International Journal of Signal Processing, Image Processing and Pattern Recognition Vol. 7, No. 6 (2014), pp. 41-50.

[5]. MVHAMMAD HAMMAD ME MON!, JIAN-PING LI!, IMRAN MEMON, RIAZ AHMED SHAIKW and FARMAN ALIMANGI," EFFICIENT OBJECT IDENTIFICATION AND MULTIPLE REGIONS OF INTEREST USING CBIR BASED ON RELATIVE LOCATIONS AND MATCHING REGIONS", 2015 IEEE, pp: 247-250.

[6]. Pushpalatha S. Nikkam, Dr. Nagaratna P. Hegde and Dr. B. Eswar Reddy," Decomposition-Based Shape Template Matching for CBIR System", 2015 IEEE International Conference on Computational Intelligence and Computing Research.

[7]. CHAWKI Youness, EL ASNAOUI Khalid, OUANAN Mohammed and AKSASSE Brahim," CBIR using the 2-D ESPRIT Method: Application to Coil_100 Database", 2015 IEEE.

[8]. Ekta Gupta and Rajendra Singh Kushwah," Combination of Global and Local Features using DWT with SVM for CBIR", 2015 IEEE.

[9]. KAMLESH KUMAR, JIAN-PING LI and ZAIN-UL-ABIDIN," COMPLEMENTARY FEATURE EXTRACTION APPROACH IN CBIR",2015 IEEE, pp: 192-197.

[10]. Vrushali A. Wankhede and Prakash S. Mohod," Content-based Image Retrieval from Videos using CBIR and ABIR algorithm", 2015 IEEE, pp: 767-771.

[11]. Radu Andrei Stefan, Ildikó-Angelica Szöke and Stefan Holban,” Hierarchical clustering techniques and classification applied in Content Based Image Retrieval (CBIR)", 10th Jubilee IEEE International Symposium on Applied Computational Intelligence and Informatics - May 21-23, 2015 - Timişoara, Romania, pp: 147-152.

[12]. Satish Tunga, D. Jayadevappa and C. Gururaj," A Comparative Study of Content Based Image Retrieval Trends and Approaches", International Journal of Image Processing (IJIP), Volume (9) : Issue (3) : 2015, pp: 127-155.

[13]. KattaSugamya,SureshPabboju, Dr.A.VinayaBabu, "A CBIR CLASSIFICATION USING SUPPORT VECTOR MACHINES" 978-1-4673-8810-8/16/\$31.00 @2016 IEEE

[14]. Syntyche Gbèhounou, François Lecellier, Christine Fernandez-Maloigne, "Evaluation of local and global descriptors for emotional impact recognition". 2016 Elsevier

[15]. Shu Wang, Jian Zhang, Senior Member, IEEE, Tony X. Han, Member, IEEE, and Zhenjiang Miao, Member, IEEE, "Sketch-Based Image Retrieval Through Hypothesis-Driven Object Boundary Selection With HLR Descriptor", vol. 17, no. 7, pp 1045-1057, july 2015.

[16]. K. Bozas and E. Izquierdo, "Large scale sketch based image retrieval using patch hashing," Adv. Visual Comput., vol. 7431, pp. 210-219, 2012.

[17]. R. Zhou, L. Chen, and L. Zhang, "Sketch-based image retrieval on a large scale database," in Proc. 20th ACM Int. Conf. Multimedia, 2012,pp. 973-976.

[18]. M. Eitz, K. Hildebrand, T. Boubekeur, and M. Alexa, "A descriptor for large scale image retrieval based on sketched feature lines," in Proc. 6th Eurograph. Symp. Sketch-Based Interfaces Modeling, 2009, pp. 29-36. 